\title{
Effect of Asymmetric Directional Spreading on the Total Radiation Stress
}

\author{
Annika O'Dea ${ }^{1}$ and Merrick C. Haller, M.ASCE ${ }^{2}$
}

\begin{abstract}
The process of wave refraction in nearshore zones can result in an asymmetric directional distribution of spectral energy when oblique, multi-directional wave fields propagate into shallow water areas. Here the effect of this directional asymmetry on the net wave-induced radiation stresses is analyzed. The net radiation stresses are calculated for a shoaled JONSWAP frequency spectrum with both symmetric and asymmetric directional distributions and then compared with those calculated from the commonly-used monochromatic formulation (appropriate for spectra that are narrow-banded in frequency and directions). Past studies have demonstrated that the use of the monochromatic approximation in radiation stress calculations results in a significant overestimation of the radiation stress components $S_{x x}$ and $S_{x y}$ in broad-banded seas. The present results show that the inclusion of directional asymmetry in radiation stress calculations reduces this overestimation for $S_{x x}$ but increases the overestimation for $S_{x y}$ for a range of dominant wave directions. Thus, directional asymmetry is an additional factor that contributes to overestimation when the monochromatic approximation is used for $S_{x y}$ and can therefore also lead to an overestimation of associated parameters such as alongshore current velocities and alongshore sediment transport rates. DOI: 10.1061/(ASCE)WW.1943-5460.0000305. ( 2015 American Society of Civil Engineers.
\end{abstract}

Author keywords: Radiation stress; Directional asymmetry; Refraction; Random waves.

\section{Introduction}

Radiation stresses, defined as the excess flow of momentum due to the presence of waves (Longuet-Higgins and Stewart 1964), are important drivers behind the cross-shore and longshore forcing that results in wave setup, set-down, and longshore currents (e.g. Svendsen 2006). Longshore currents entrain and transport sediment and therefore play an important role in short- and long-term coastal evolution (Komar 1998). For a real wave field, with the total energy being a linear combination of a number of components distributed in frequency and direction, the offdiagonal radiation stress component $S_{x y}$ is defined by

$$
S_{x y}=\rho g \int_{0}^{\infty} \int_{-\pi}^{\pi} E(f, \theta) \frac{c_{g}(f)}{c(f)} \sin (\theta) \cos (\theta) d \theta d f
$$

where $\rho=$ density of seawater; $g=$ acceleration of gravity; $c_{g}(f)=$ frequency-dependent group velocity; $c(f)=$ frequencydependent phase velocity (both velocities given by linear theory); $\theta=$ wave angle of approach; and $E(f, \theta)=$ frequency-directional energy spectrum (in units of $\mathrm{m}^{2} \mathrm{~s} / \mathrm{rad}$ ) (Battjes 1972). Eq. (1) (referred to as the true formulation hereinafter) requires knowledge of the full frequency and directional distribution of spectral energy, which is not always available in practice. For this reason, the monochromatic radiation stress formulation of reduced form

\footnotetext{
${ }^{1}$ Graduate Research Assistant, School of Civil and Construction Engineering, 220 Owen Hall, Oregon State Univ., Corvallis, OR 97331.

${ }^{2}$ Associate Professor, School of Civil and Construction Engineering, 220 Owen Hall, Oregon State Univ., Corvallis, OR 97331 (corresponding author). E-mail: merrick.haller@oregonstate.edu

Note. This manuscript was submitted on October 2, 2014; approved on February 6, 2015; published online on June 10, 2015. Discussion period open until November 10, 2015; separate discussions must be submitted for individual papers. This technical note is part of the Journal of Waterway, Port, Coastal, and Ocean Engineering, (C) ASCE, ISSN 0733-950X/ 06015004(6)/\$25.00.
}

given by (Longuet-Higgins 1970a, b)

$$
S_{x y}=\frac{1}{8} \rho g H^{2} \frac{c_{g}}{c} \sin (\theta) \cos (\theta)
$$

is typically used where $H=$ wave height determined from the total energy; and the values of group velocity, $c_{g}$ and phase velocity, $c$, are taken at the peak frequency. The dominant wave angle of approach, $\theta$, is often taken as the peak or energyweighted direction at the peak frequency. If the spectrum is narrow-banded in frequency and directional space, the difference between the monochromatic formulation and the true formulation is minimal.

The accuracy of this narrow-banded (monochromatic) radiation stress approximation has been the focus of several studies (Battjes 1972; Freilich and Pawka 1987; Feddersen 2004) since the radiation stress formulation was first proposed. Battjes (1972) conducted an analytic analysis of the ratio of total energy to the radiation stress using the Pierson-Moskowitz (PM) frequency spectrum and several symmetric directional spreading functions. He determined that the spreading of energy over a range of frequencies did not significantly affect the ratio of energy to radiation stress, but that high directional spreading (or short-crestedness) did have an effect on the ratio. That study found that the use of the narrow-banded approximation for the $S_{x x}$ and $S_{x y}$ components in short-crested seas significantly overestimated the actual radiation stress.

In a more recent study using field data observed at $8 \mathrm{~m}$ water depth on the Outer Banks, NC, where broad-banded sea states are common, Feddersen (2004) confirmed that the narrow-banded estimates of $S_{x x}$ and $S_{x y}$ radiation stress components are systematically larger than the true radiation stress values calculated from the full frequency-directional spectrum, and quantified the dependence of this difference on the directional spread. Also, using a PM frequency spectrum and a symmetric directional spreading function similar to the method used by Battjes (1972), Feddersen (2004) developed a parameterization for including the effects of 
directional spreading into the calculation of the $S_{x x}$ and $S_{x y}$ radiation stress components.

The studies mentioned above demonstrated that the use of the narrow-banded radiation stress approximations can result in biased radiation stress values and consequently biased longshore current and sediment transport values. However, neither study considered the effect of an asymmetric directional distribution. A recent study by Lee et al. (2010) indicated that the symmetric directional spreading function is less appropriate in shallow water, where radiation stress forcing is most important. Lee et al. did not analyze the relationship between directional asymmetry and radiation stresses, but they demonstrated that wave refraction in shallow water results in an asymmetric directional distribution of spectral energy, and proposed a modification to a commonly used directional spreading function to parameterize this asymmetry. Here the asymmetric spectral formulation proposed by Lee et al. (2010) to investigate the impact of an asymmetric directional distribution on the true radiation stress values is used.

The main objective of this study is to analyze the effect of asymmetry in the directional distribution of spectral energy on calculations of wave-induced radiation stresses. The wave-induced radiation stresses are determined using (1) a narrow-banded approximation, (2) a JONSWAP frequency spectrum with a symmetric directional spreading function, and (3) a JONSWAP frequency spectrum with an asymmetric directional spreading function, and the resulting values are compared. Basic conclusions about the importance of directional asymmetry in nearshore radiation stress calculations are made, and the implications of the use of narrowbanded approximations or symmetric spectra in the calculation of radiation stress for coastal engineering projects are discussed.

\section{Methods}

\section{Frequency-Directional Wave Spectrum}

The frequency-directional wave spectrum $E(f, \theta)$ is a product of the frequency spectrum $S(f)$ and the directional spreading function $G(f, \theta)$, shown in Eq. (3)

$$
E(f, \theta)=S(f) G(f, \theta)
$$

where $E(f, \theta)$ is in units of $\mathrm{m}^{2} \mathrm{~s} / \mathrm{rad}$. The frequency-directional wave spectrum was calculated using both symmetric and asymmetric directional spreading functions.

\section{Directional Spreading Function-Symmetric Spectrum}

For the symmetric directional spectrum, the Longuet-Higgins et al. (1961) formulation for the directional spreading function was used, where the directional spreading function $G(f, \theta)$ can be written as

$$
G(f, \theta)=G_{O} \cos ^{2 s}\left(\frac{\theta-\theta_{p}}{2}\right)
$$

where

$$
\begin{gathered}
G_{O}=\left[\int_{\theta_{\min }}^{\theta_{\max }} \cos 2 s\left(\frac{\theta-\theta_{p}}{2}\right)\right]^{-1} \\
s=\left\{\frac{s_{\max } \cdot\left(f / f_{p}\right)^{5}, f \leq f_{p}}{s_{\max } \cdot\left(f / f_{p}\right)^{-2.5}, f \geq f_{p}}\right.
\end{gathered}
$$

where $\theta_{p}$ is the peak direction, $\theta_{\min }$ and $\theta_{\max }$ are the maximum and minimum angle of interest, and $f_{p}$ is the peak frequency. The spreading parameter $s$ was developed by Goda and Suzuki (1975).

\section{Directional Spreading Function-Asymmetric Spectrum}

As multi directional wave fields propagate in intermediate to shallow water depths, each directional component will refract at a different rate, resulting in an asymmetric distribution of spectral energy around the peak wave direction. A model function for this asymmetric distribution was given by Lee et al. (2010). In the asymmetric case, the spreading function $G_{A}(f, \theta)$ is given by:

$$
\begin{gathered}
G_{A}(f, \theta)=G_{O A} \cos 2 s\left(\frac{\theta-\theta_{p}}{2} \xi\right) \\
G_{O A}=\left[\int_{\theta_{\min }}^{\theta_{\max }} \cos ^{2 s}\left(\frac{\theta-\theta_{p}}{2} \xi\right)\right]^{-1} \\
\xi=\left[\begin{array}{l}
\exp (-\mu), \theta \geq \theta_{p} \\
\exp (+\mu), \theta \leq \theta_{p}
\end{array}\right]
\end{gathered}
$$

where $\mu=$ asymmetry parameter and $s$ is given in Eq. (5).

When $\mu$ is 0 (as would be the case if the peak wave direction were shore normal), $\xi$ is equal to 1 and the asymmetric directional spreading function defined above collapses into the symmetric directional spreading function proposed by Longuet-Higgins et al. (1961). For a set of obliquely incident deep water conditions, Lee et al. found $\mu$ and $s_{\max }$ as a function of relative depth $h / L_{p o}$, where $h$ is the water depth and $L_{p o}$ is the offshore peak wavelength. To determine $\mu$ and $s_{\max }$, Lee et al. (2010) decomposed the wave spectrum into frequency and directional components in deep water, and then shoaled and refracted each component across a uniform sloping bathymetry. The authors then found the values of $\mu$ and $s_{\max }$ that led to the best fit of the transformed nearshore wave spectrum for all water depths. To determine asymmetry parameters for a different set of incident deep water conditions, this process would need to be repeated (or a parameterization developed). The asymmetric directional spreading function was then validated against spectra computed from field data in $8 \mathrm{~m}$ water depth from Duck, North Carolina (same field site as Feddersen 2004).

Here the authors use the $\mu$ and $s_{\max }$ values found by Lee et al. for each set of input conditions in all calculations. Both a high directional spread case $\left(s_{\max }=10\right)$ and a low directional spread case $\left(s_{\max }=75\right)$ were considered. The offshore $s_{\max }$ and $\theta_{p o}$ values along with the $\mu$ and $s_{\max }$ values at the $10 \mathrm{~m}$ contour line are listed in Table 1. Also following Lee et al., the directional limits $\theta_{\min }$ and $\theta_{\max }$ were then given by the following equations:

$$
\theta_{\min }=\theta_{p}-\frac{2 \pi}{1+\exp (2 \mu)}, \theta_{\max }=\theta_{p}+\frac{2 \pi}{1+\exp (-2 \mu)}
$$

For further discussion of the asymmetric directional spreading function, see Lee et al. (2010).

\section{Frequency Spectrum}

For the JONSWAP frequency spectrum (Hasselmann et al. 1973), $S(f)$ is given by

$$
S(f)=\beta_{J} H_{s}^{2} T_{P}^{-4} f^{-5} \exp \left[-1.25\left(T_{P} f\right)^{-4}\right] \gamma^{\exp \left[-\left(T_{P} f-1\right)^{2} / 2 \sigma^{2}\right]}
$$


where $f=$ frequency, $H_{s}=$ significant wave height, $T_{p}=$ peak period, $\gamma=$ peak enhancement parameter, and with

$$
\begin{gathered}
B_{J} \cong \frac{0.0594}{0.23+0.0336 \gamma-0.185(1.9+\gamma)^{-1}}[1.094-0.01915 \ln \gamma] \\
\sigma=\left\{\frac{\sigma_{a}=0.07: f \leq f_{p}}{\sigma_{b}=0.09: f \geq f_{p}}\right. \\
T_{P} \cong \frac{T_{1 / 3}}{\left[1-0.132(\gamma+0.2)^{-0.559}\right]}
\end{gathered}
$$

where $T_{s}=$ significant wave period. A peak enhancement parameter value of $\gamma=3.3$ was used in all trials, and the spectra were calculated at the $10 \mathrm{~m}$ contour line.

\section{Input Conditions}

An offshore significant wave height $H_{s o}$ of $5 \mathrm{~m}$ and a significant period $T_{s}$ of $10 \mathrm{~s}$ were used in all calculations along with three offshore peak wave angles $\left(\theta_{p o}=0^{\circ}, 30^{\circ}\right.$, and $\left.60^{\circ}\right)$ and two directional spreading values (offshore $s_{\max }=10$ and 75). The subscript $o$ indicates offshore conditions in all cases. The radiation stresses were then calculated for $10 \mathrm{~m}$ water depth. The significant wave height $H_{s}$ and peak wave direction $\theta_{p}$ at $10 \mathrm{~m}$ depth were calculated from the offshore conditions using linear shoaling and Snell's Law. Input conditions were chosen to match those in the Lee et al. (2010), and the spreading parameter $s_{\max }$ and asymmetry parameter $\mu$ suggested by Lee et al. for each set of conditions were used in the directional spreading function calculations [See Lee et al. (2010), Figs. 5 and 6, respectively]. Input conditions for all trials are given in Table 1.

\section{Radiation Stress}

The radiation stress tensor $S_{i j}$ was then numerically determined using (1) a narrow-banded approximation, and the full frequencydirectional spectrum for (2) a symmetric directional distribution, and (3) an asymmetric directional distribution. Adopting the

\begin{tabular}{|c|c|c|c|c|}
\hline $\begin{array}{l}\text { Directional } \\
\text { distribution }\end{array}$ & Offshore $\theta\left(^{\circ}\right)$ & $\begin{array}{c}\text { Offshore } \\
s_{\max }\end{array}$ & $\begin{array}{l}\text { Asymmetry } \\
\text { parameter } \mu\end{array}$ & $\begin{array}{c}s_{\max } \text { at } \\
10 \mathrm{~m} \\
\text { water } \\
\text { depth }\end{array}$ \\
\hline \multirow[t]{6}{*}{ Asymmetric } & 0 & 10 & 0 & 55 \\
\hline & 30 & 10 & -0.28 & 69 \\
\hline & 60 & 10 & -0.48 & 150 \\
\hline & 0 & 75 & 0 & 330 \\
\hline & 30 & 75 & -0.14 & 400 \\
\hline & 60 & 75 & -0.25 & 1,000 \\
\hline \multirow[t]{6}{*}{ Symmetric } & 0 & 10 & 0 & 55 \\
\hline & 30 & 10 & 0 & 68 \\
\hline & 60 & 10 & 0 & 120 \\
\hline & 0 & 75 & 0 & 330 \\
\hline & 30 & 75 & 0 & 400 \\
\hline & 60 & 75 & 0 & 1,000 \\
\hline
\end{tabular}
notation of Feddersen (2004), the radiation stress formulations for

Table 1. Input Conditions for All Trials

Note: An offshore significant wave height $H_{s o}$ of $5 \mathrm{~m}$ and a significant period $T_{p}$ of $10.7 \mathrm{~s}$ were used in all calculations. An increase in $s_{\max }$ implies a decrease in directional spread. narrow-banded (nb) seas can be rewritten as (Svendsen 2006)

$$
\begin{gathered}
S_{x x}{ }^{(\mathrm{nb})}=\frac{1}{8} \rho g H_{\mathrm{RMS}}{ }^{2}\left[n\left(\cos ^{2} \theta+1\right)-\frac{1}{2}\right] \\
S_{y y}{ }^{(\mathrm{nb})}=\frac{1}{8} \rho g H_{\mathrm{RMS}}{ }^{2}\left[n\left(\sin ^{2} \theta+1\right)-\frac{1}{2}\right] \\
S_{x y}{ }^{(\mathrm{nb})}=S_{y x}{ }^{(\mathrm{nb})}=\frac{1}{16} \rho g H_{\mathrm{RMS}}{ }^{2} n \sin 2 \theta
\end{gathered}
$$

where $H_{\mathrm{RMS}}=$ root mean squared wave height; $\theta=$ wave direction at the peak frequency; $k=$ wave number (corresponding to the peak frequency); and $h=$ water depth, and with $n$ given by

$$
n=\frac{C_{g}}{C}=\frac{1}{2}\left(1+\frac{2 k h}{\sinh (2 k h)}\right)
$$

If the full frequency-directional spectrum is known, the true radiation stress (tr) can be calculated from the extended equations given by:

$$
\begin{aligned}
& S_{x x}{ }^{(\mathrm{tr})}=\rho g \int_{0}^{\infty} \int_{\theta_{\min }}^{\theta_{\max }} E(f, \theta)\left[n(f)\left(\cos ^{2} \theta+1\right)-\frac{1}{2}\right] d \theta d f \\
& S_{y y}{ }^{(\mathrm{tr})}=\rho g \int_{0}^{\infty} \int_{\theta_{\min }}^{\theta_{\max }} E(f, \theta)\left[n(f)\left(\sin ^{2} \theta+1\right)-\frac{1}{2}\right] d \theta d f \\
& S_{x y}{ }^{(\mathrm{tr})}=S_{y x}{ }^{(\mathrm{tr})}=\rho g \int_{0}^{\infty} \int_{\theta_{\min }}^{\theta_{\max }} \frac{1}{2} E(f, \theta) n(f)(\sin 2 \theta) d \theta d f
\end{aligned}
$$

where $n$ is now a function of frequency. These equations, in addition to Eqs. 15-17, were used to calculate the true radiation stress values and the narrow-banded radiation stress values at $10 \mathrm{~m}$ water depth, respectively.

\section{Results and Discussion}

Examples of the difference between asymmetric and symmetric directional spreading functions are shown in Figs. 1(a and b), which shows the directional distributions at the peak frequency for cases with an offshore $s_{\max }=10$ and $\theta_{p o}=30^{\circ}$ and $60^{\circ}$, respectively. The full directional spectra are shown in Fig. 2, which demonstrates that the use of an asymmetric directional spreading function results in a visibly asymmetric wave spectrum.

The wave-induced radiation stresses were calculated using the three previously described methods, and the resulting values for the narrow-banded formulation are shown in Table 2. Fig. 3 shows the ratio of the true radiation stress to the narrow-banded radiation stress $\left(S_{x x}{ }^{(\mathrm{tr})} / S_{x x}{ }^{(\mathrm{nb})}\right.$ and $\left.S_{x y}{ }^{(\mathrm{tr})} / S_{x y}{ }^{(\mathrm{nb})}\right)$. Where the ratios are closest to 1.0 the narrow-banded approximation works well, ratios significantly different from one indicate the deficiencies in the narrow-banded approximation. The use of the full frequency-direction spectrum (symmetric or asymmetric) in the true radiation stress calculations resulted in a decrease in the components $S_{x x}$ and $S_{x y}$ from that calculated using the narrow-banded approximation for all wave angles included in the analysis (excluding $S_{x y}$ with $\theta_{p o}=0^{\circ}$, in which case all values were 0 ). Although the peak wave direction is the same with both the symmetric and asymmetric directional spreading function, the use of an asymmetric directional spreading function results in a mean wave direction closer to shore normal, thereby increasing the value of $S_{x x}$. Thus the narrow-banded approximation is less of an under estimation for this component. However, for the $S_{x y}$ component, the effect is opposite in that including directional asymmetry further reduces 

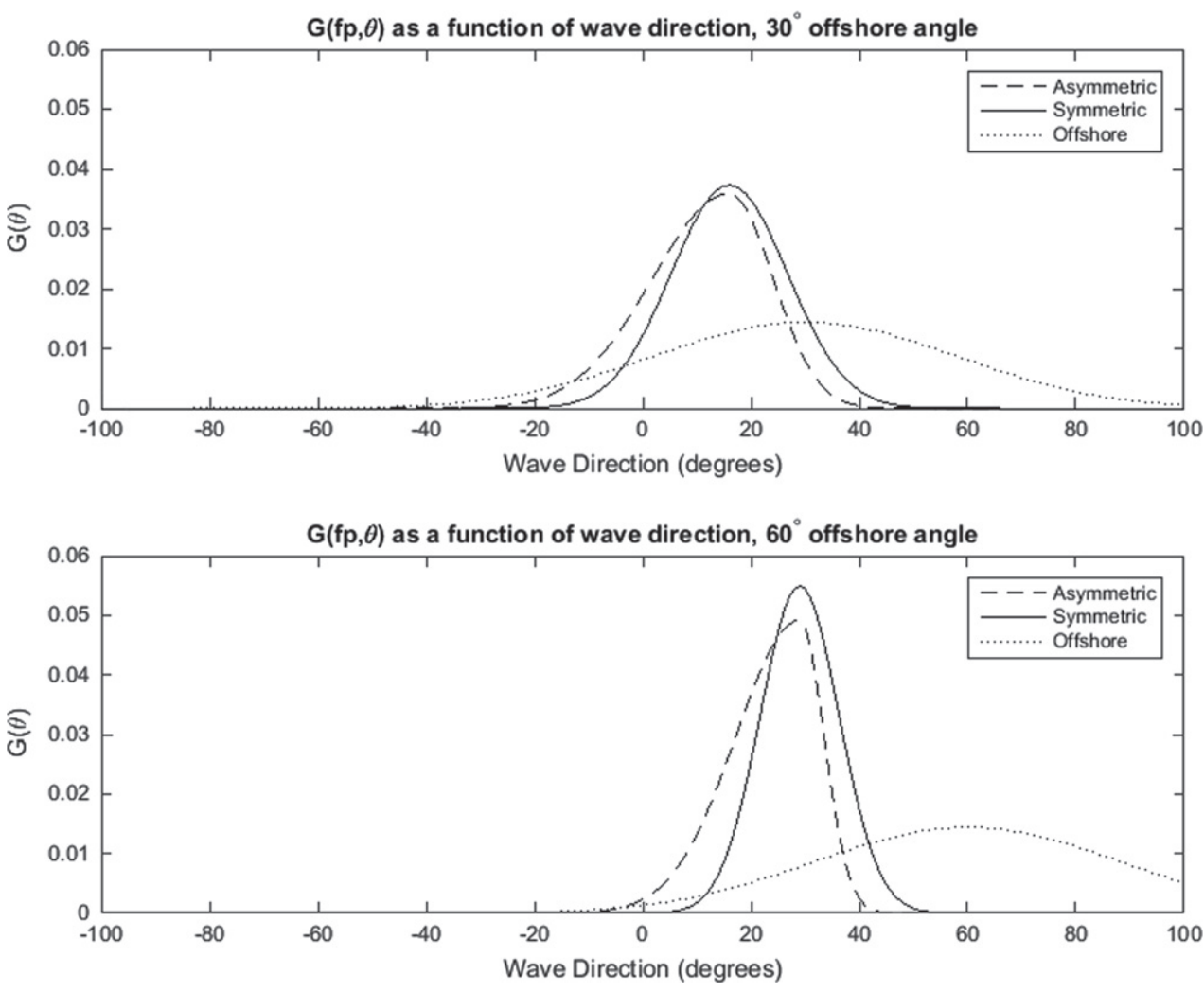

Fig. 1. $G(f, \theta)$ at the peak frequency $\left(f_{p}=0.0934\right)$ from trials with high directional spread (offshore $s_{\max }=10$ ) and offshore peak wave direction of (a) $30^{\circ}$; and (b) $60^{\circ}$. Offshore incident directional distribution (symmetric) shown as dotted lines
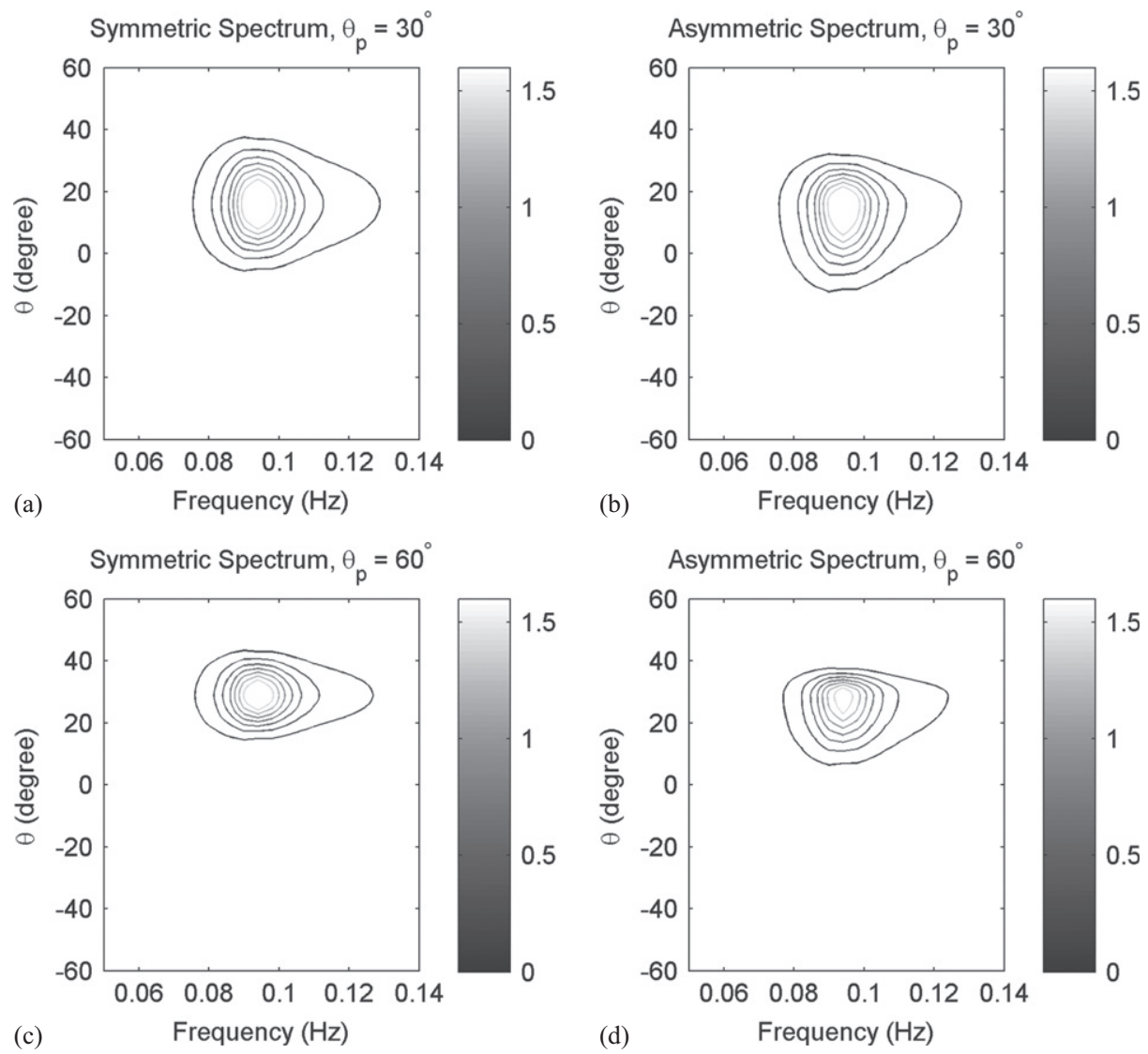

Fig. 2. Frequency directional spectra $S(f, \theta)\left(\mathrm{m}^{2} / \mathrm{Hz} / \mathrm{deg}\right)$ from cases with (a) symmetric spectrum, $30^{\circ}$ offshore wave direction; (b) asymmetric spectrum, $30^{\circ}$ offshore wave direction; (c) symmetric spectrum, $60^{\circ}$ offshore wave direction; and (d) asymmetric spectrum, $60^{\circ}$ offshore wave direction for high directional spreading (offshore $s_{\max }=10$ ) 
Table 2. Radiation Stress Values at $10 \mathrm{~m}$ Water Depth for All Trials

\begin{tabular}{lccccccc}
\hline $\begin{array}{l}\text { Offshore } \\
\theta_{p o}\left({ }^{\circ}\right)\end{array}$ & $\begin{array}{c}\text { Offshore } \\
S_{\max }\end{array}$ & $\begin{array}{c}S_{x x}{ }^{(\mathrm{nb})} / \rho \\
\left(\mathrm{m}^{3} / \mathrm{s}^{2}\right)\end{array}$ & $\begin{array}{c}S_{x x}{ }^{(\mathrm{tr} S)} / \rho \\
\left(\mathrm{m}^{3} / \mathrm{s}^{2}\right)\end{array}$ & $\begin{array}{c}S_{x x}{ }^{(\mathrm{tr} A)} / \rho \\
\left(\mathrm{m}^{3} / \mathrm{s}^{2}\right)\end{array}$ & $\begin{array}{c}S_{x y}{ }^{(\mathrm{nb})} / \rho \\
\left(\mathrm{m}^{3} / \mathrm{s}^{2}\right)\end{array}$ & $\begin{array}{c}S_{x y}{ }^{(\mathrm{tr} S)} / \rho \\
\left(\mathrm{m}^{3} / \mathrm{s}^{2}\right)\end{array}$ \\
\hline 0 & 10 & 19.78 & 17.71 & 17.71 & 0.00 & 0.00 & $\begin{array}{c}S_{x y}{ }^{(\mathrm{tr} A)} / \rho \\
\left(\mathrm{m}^{3} / \mathrm{s}^{2}\right)\end{array}$ \\
30 & 10 & 16.89 & 15.29 & 15.68 & 3.29 & 2.82 & 0.00 \\
60 & 10 & 9.44 & 8.71 & 9.31 & 3.33 & 3.02 & 2.90 \\
0 & 75 & 19.78 & 18.37 & 18.37 & 0.00 & 0.00 & 0.00 \\
30 & 75 & 16.89 & 15.70 & 15.82 & 3.29 & 3.08 & 3.15 \\
60 & 75 & 9.44 & 8.79 & 8.93 & 3.33 & 3.05 \\
\hline
\end{tabular}
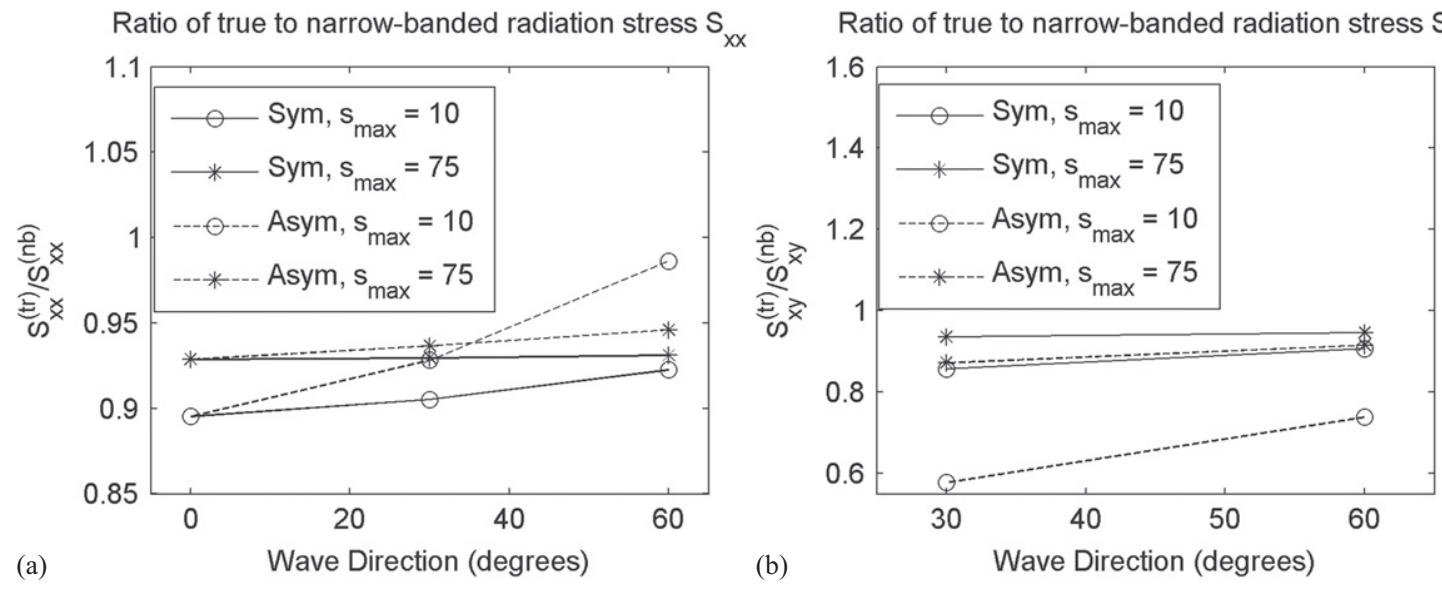

Fig. 3. Ratio of true to narrow-banded radiation stresses (a) $S_{x x}{ }^{(\mathrm{tr})} / S_{x x}{ }^{(\mathrm{nb})}$ : and (b) $S_{x y}{ }^{(\mathrm{tr})} / S_{x y}{ }^{(\mathrm{nb})}$ as a function of dominant offshore wave direction for high (offshore $s_{\max }=10$ ) and low (offshore $s_{\max }=75$ ) directional spreading

the value beyond the symmetric directional spread value. Hence, the narrow-banded approximation is an even greater over-estimate than previously thought.

For the $S_{x x}$ component, the symmetric wave spectrum resulted in the lowest value in each case, with the percent change from the narrow-banded radiation stress value decreasing with increasingly oblique offshore wave angles but increasing with directional spread (with a maximum percent change of $10.5 \%$ in trials with high directional spread versus $7.1 \%$ from trials with low directional spread, both with $\theta_{p o}=0^{\circ}$ ). When an asymmetric wave spectrum was used, the percent decrease from the narrow-banded approximation was less than when a symmetric spectrum was used (except when waves were approaching shore normally, in which case the two formulations were identical), with the magnitude of change being a function of both directional spread and offshore wave angle [Fig. 3(a)].

Similar to the results presented by Feddersen (2004) and Battjes (1972), there is a bigger discrepancy in the off-diagonal radiation stress component $S_{x y}$ when using a narrow-banded assumption versus using the full directional spectrum. In addition, this discrepancy is always larger when the directional distribution is asymmetric. The largest percent change was seen in trials with $\theta_{p o}=30^{\circ}$ and high directional spread (42.3\% decrease). In contrast, for a symmetric directional distribution the percent change from the narrow-banded approximation was only $14.3 \%$. Trials with low directional spread resulted in full-spectral $S_{x y}$ values closer to the narrow-banded approximation for all sets of input conditions.

The amount of directional spread in spectral formulations is controlled by the $s$ parameter in the directional spreading function [shown in Eq. (5)]. In the present study, two offshore $s_{\max }$ values were assessed, a high-spreading case $\left(s_{\max }=10\right)$ and a lowspreading case $\left(s_{\max }=75\right)$, which were selected to be consistent with the conditions analyzed by Lee et al. (2010) and representative of a range of field conditions. As Battjes (1972) and Feddersen (2004) demonstrated, the difference between the true radiation stress value and the narrow-banded approximation is dependent on the directional spread in the wave spectrum. The present results show that asymmetry in the directional distribution is also of importance to the radiation stress calculation. The presence of directional asymmetry was found to mitigate the overestimation of $S_{x x}$ that occurs when the narrow-banded approximation is used, especially for higher directional spreading values. The effect of directional asymmetry also increased with directional spreading for the calculation of $S_{x y}$.

Past studies have demonstrated that the narrow-banded approximation leads to an overestimate of the radiation stresses $S_{x x}$ and $S_{x y}$ owing to the effects of directional spreading (Battjes 1972; Ruessink et al. 2001; Feddersen 2004; Puleo 2010). In addition, the magnitude of $S_{x y}$ outside the surf zone has been both theoretically and empirically linked to the maximum longshore current velocity (e.g. Guza et al. 1986; Feddersen et al. 1998), and the importance of the inclusion of directional spreading in alongshore current and sediment transport calculation has been discussed in a number of studies (Ruessink et al. 2001; Puleo 2010; Barbaro et al. 2014). The present results also indicate that asymmetry in the directional distribution induced by wave refraction has a significant effect on the net radiation stresses. In fact, accounting for directional asymmetry is just as important as accounting for directional spreading in the accurate estimation of $S_{x y}$.

\section{Conclusions}

Gradients in wave radiation stresses are important drivers of many nearshore processes. To facilitate their calculation and use, radiation stresses are often approximated using a monochromatic radiation stress formulation, under the assumption that the sea state is narrow- 
banded in frequency and directional space and therefore the difference between the monochromatic formulation and that given by using the full frequency-direction spectrum for real seas is expected to be small. However, past studies have shown that the narrowbanded approximation leads to errors that correlate with the level of directional spreading in the spectrum. In this study, the importance of refraction-induced directional asymmetry on the radiation stress calculations was also investigated. Radiation stresses calculated using the standard narrow-banded approximation were compared with those calculated using the full frequency-direction spectrum where the full spectrum included a JONSWAP frequency spectrum with either a symmetric or asymmetric directional spreading function. Results show that the presence of directional asymmetry leads to a smaller error in the $S_{x x}$ value with the narrow-banded approximation, but increases the error in the $S_{x y}$ value from the narrowbanded approximation. Essentially, directional asymmetry owing to refraction will skew energy toward less oblique directions. This will increase the true value of $S_{x x}$, making the narrow-banded value less of an overestimate, and it will decrease the true value of $S_{x y}$, making the narrow-banded value even more of an overestimate. Additionally, the effect of directional asymmetry was found to increase with overall directional spread and to be of the same order as the effect of directional spreading as a whole for $S_{x y}$. These results suggest that the use of a narrow-banded radiation stress approximation in nearshore models could result in a significant overestimate of alongshore current velocities and longshore sediment transport rates, particularly in broad-banded seas.

\section{References}

Barbaro, G., Foti, G., Sicilia, L., and Malara, G. (2014). "A formula for the calculation of the longshore sediment transport including spectral effects." J. Coast. Res., 30(5), 961-966.

Battjes, J. A. (1972). "Radiation stress in short-crested waves." J. Mar. Res., 30(1), 56-64.

Feddersen, F. (2004). "Effect of wave directional spread on radiation stress: Comparing theory and observations." Coast. Eng., 51(5-6), 473-481.
Feddersen, F., Guza, R. T., Elgar, S., and Herbers, T. H. C. (1998). "Alongshore momentum balances in the nearshore." J. Geophys. Res., 103(C8), 15667-15676.

Freilich, M. H., and Pawka, S. S. (1987). "Statistics of $\mathrm{S}_{x y}$ estimates." J. Phys. Oceanogr., 17(10), 1786-1797.

Goda, Y., and Suzuki, Y. (1975). "Computation of refraction and diffraction of sea waves with Mitsuyasu's directional spectrum." Rep. No. 230, The Port and Harbour Research Institute, Japan (in Japanese).

Guza, R. T., Thorton, E. B., and Christensen, N., Jr. (1986). "Observations of steady longshore currents in the surf zone." J. Phys. Oceanogr., 16(11), 1959-1969.

Hasselmann, K., et al. (1973). "Measurements of wind-wave growth and swell decay during the joint north sea wave project (JONSWAP)." Deutches Hydrographisches Zeitschrift, 8(12), 1-95.

Komar, P. D. (1998). Beach processes and sedimentation, 2nd Ed., Prentice-Hall, Upper Saddle River, NJ, 9.

Lee, C., Jung, J. S., and Haller, M. C. (2010). “Asymmetry in directional spreading function of random waves due to refraction." J. Waterway, Port, Coastal, Ocean Eng., 10.1061/(ASCE)WW.1943-5460.0000006, $1-9$.

Longuet-Higgins, M. S. (1970a). "Longshore currents generated by obliquely incident sea waves, 1." J. Geophys. Res., 75(33), 6778-6789.

Longuet-Higgins, M. S. (1970b). "Longshore currents generated by obliquely incident sea waves, 2." J. Geophys. Res., 75(33), 6791-6801.

Longuet-Higgins, M. S., Cartwright, D. E., and Smith, N. D. (1961). "Observations of the directional spectrum of sea waves using the motion of a floating buoy." Proc., Conf. on Ocean Wave Spectra, Prentice-Hall, Englewood Cliffs, NJ, 111-136.

Longuet-Higgins, M. S., and Stewart, R. W. (1964). "Radiation stresses in water waves; A physical discussion with applications." Deep-Sea Res., 11(4), 529-562.

Puleo, J. (2010). "Estimating alongshore sediment transport and the nodal point location on the Delaware-Maryland Coast." J. Waterway, Port, Coastal, Ocean Eng., 10.1061/(ASCE)WW.1943-5460.0000034, 135-144.

Ruessink, B. G., Miles, J. R., Feddersen, F., Guza, R. T., and Elgar, S. (2001). "Modeling the alongshore current on barred beaches." J. Geophys. Res., 106(C10), 22451-22463.

Svendsen, I. A. (2006). Introduction to nearshore hydrodynamics, World Scientific Publishing Co. Pte. Ltd., Singapore, 11-12. 